\title{
Evaluation of Mixture Magnesium Oxide and Zinc Oxide Nanoparticles against Multi-drug-resistance Mycobacterium tuberculosis by Microplate Alamar Blue Assay
}

\section{Shahriari}

Guilan University of Medical Sciences

Alireza Jafari ( $\square$ Dr.alireza.jafarii@@gmail.com )

Inflammatory Lung Disease Research Center https://orcid.org/0000-0001-8545-4324

F. Movahedzadeh

University of Illinois at Chicago College of Medicine

A.A. Foumani

Guilan University of Medical Sciences

S. Falahatkar

Guilan University of Medical Sciences

\section{Research note}

Keywords: Nanoparticle; MDR-TB; Magnesium oxide; Zinc oxide; Mycobacterium tuberculosis

Posted Date: August 24th, 2019

DOI: https://doi.org/10.21203/rs.2.13541/v1

License: (c) (1) This work is licensed under a Creative Commons Attribution 4.0 International License.

Read Full License 


\section{Abstract}

Objective The current study is the first experimental study of which has evaluated the MICs and MBCs of ZnONPs, MgONPs, and mixture MgONPs-ZnONPs against $\mathrm{H} 37 \mathrm{Rv}$ Mtb and MDR-Mtb.Results The MIC of MgONPs and ZnONPs were 0.195 and $0.468 \mu \mathrm{g} . \mathrm{ml}-1$ against 104 of H $37 \mathrm{Rv}$ Mtb. As well, $0.166 \mu \mathrm{g} . \mathrm{ml}-1$ of MgONPs-ZnONPs was able to inhibit $10-4$ H 37 Rv Mtb. The MIC of MgONPs against 104 concentrations of MDR-Mtb was $12.5 \mu \mathrm{g} . \mathrm{ml}-1$. The MIC of MgONPs/ZnONPs against 104 concentrations of MDR-Mtb reached to $0.664 \mu \mathrm{g} . \mathrm{ml}-1$. Based on the results, the MBC value of ZnONPs increased to $1.875 \mu \mathrm{g} \cdot \mathrm{ml}-1$ against $10-4$ concentrations of MDR-Mtb. Testing showed that the MBCs of MgONPs/ZnONPs reached to $1.328 \mu \mathrm{g} . \mathrm{ml}-1$ against 104 concentrations of MDR-Mtb. The half maximal inhibitory concentration (IC50) against MDR-TB was $0.779 \mu \mathrm{g} . \mathrm{ml}-1$ for ZnONPs and $0.883 \mu \mathrm{g} . \mathrm{ml}-1$ for MgONPs-ZnONPs. The MgONPs-ZnONPs was not toxic to Vero cell lines however ZnONPs could inhibit the Vero and HepG 2 cell lines. We found that ZnONPs and mixture MgONPs-ZnONPs not only have higher bactericide behavior but might have also synergistic effects against MDR-TB.

\section{Background}

Multi-drug-resistant tuberculosis (MDR-TB) is a form of tuberculosis (TB) infection caused by Mycobacterium tuberculosis $(\mathrm{Mtb})$ that are resistant to treatment with at least isoniazid (INH) and rifampin (RMP). It is estimated that MDR-TB caused 480,000 new TB cases and 250,000 deaths in 2015 [1].

Today, nanotechnology has attracted medical researchers for over a century and is now with heavy steps used in biomedical sciences [2]. The researchers have found, metallic NPs have shown antitubercular properties against Mtb [3]. In fact, there is a focus of interest related to using mixed metallic NPs because of their outstanding potential against MDR-TB $[2,4,5]$.

Studies have shown that in the process pathogenesis of pulmonary tuberculosis, the metallic NPs can penetrate within the calcified granuloma and can attach to Mtb and kill it. The metallic NPs can also eliminate Mtb into the macrophage without any toxicity effect. Therefore, the metallic NPs especially mixed metallic NPs have potentially capable of electrostatic interactions with the Mtb cell membrane [6].

The anti-tubercular potency of metallic NPs such as MgONPs and ZnONPs are still unknown against MDR-Mtb. It is necessary to establish consistent conditions in order to directly compare the effects of the MgONPs and ZnONPs dosages on a clinical strain of MDR-Mtb. Under the consistent conditions, we investigated and compared the effects of different dosage of mixture MgONPs and ZnONPs on two different types of clinical isolates MDR-Mtb and their standard strain. According to the search, this is the first study to use the "Microplate Alamar Blue" method as a rapid and a safety test in order to quantify and compare anti-tubercular activities of mixture MgONPs and ZnONPs against two drug-resistant strains of Mtb clinical isolates with standard strain. Moreover, this is the first time to study the MgONPsZnONPs toxicity on African green monkey (Vero) and human liver cancer $\left(\mathrm{HepG}_{2}\right)$ cell lines. 


\section{Methods}

\section{Study design and bacterial isolates}

The multidrug resistance strain of Mtb isolated from a 55-year-old man who was proven tuberculosis by imaging, clinical findings, histological and cytological observations, previously. The patient had consumed first-line anti-tubercular treatment for 6 months but the symptoms of TB had reappeared about 7 months after completing the treatment. Spectrum sample of patients was cultured in LJ medium for 28 days. The susceptibility drug testing was done by the indirect proportion method to determining of MDRMtb [7].

\section{Preparing Nanoparticles}

In this study, MgONPs (average diameter of $20 \mathrm{~nm}$ ) and ZnONPs (average diameter of $4 \mathrm{~nm}$ ) were purchased from Tehran University of Medical Sciences (Tehran, Iran). Before each experiment, MgONPs and ZnONPs were sterilized through heating in an oven at $200^{\circ} \mathrm{C}$ for one hour.

\section{Antibiotic susceptibility test}

To determine the minimum inhibition concentration (MICs) of NPs, the microplate Alamar blue (MABA) assay is used [8]. Two hundred microliter $(200 \mu \mathrm{l})$ of sterile deionized water added to outer-perimeter wells of 96-well microplates. Then, $100 \mathrm{ml}$ of 7H9 GC broth was added to each well. $100 \mu \mathrm{l}$ of MgONPs (12.5 $\mu \mathrm{g} \cdot \mathrm{ml}-1)$, ZnONPs (30 $\mu \mathrm{g} \cdot \mathrm{ml}-1)$ and MgONPs-ZnONPs $(42.5 \mu \mathrm{g} \cdot \mathrm{ml}-1)$ solutions were added to each well, and the serial dilutions were done. A hundred microliter (100 $\mu$ l) of H37Rv Mtb (Razi serum and vaccine research institute, IRAN) was added to the wells [9]. The plates incubated at $37^{\circ} \mathrm{C}$ for 5 days. Fifty microliters $(50 \mu \mathrm{l})$ of a freshly prepared $1: 1$ mixture of Alamar blue reagent and $10 \%$ Tween 80 added to well and reinsulated at $37^{\circ} \mathrm{C}$ for 24 hours. The blue color in the well interpreted as no growth and a pink color scored as growth. The MICs was defined as the lowest drug concentration which prevented a color change from blue to pink [9].

To determine the minimum bactericidal concentration (MBCs) of NPs against MDR-Mtb (Razi serum and vaccine research institute, IRAN), proportion method was also done. $10 \mathrm{ml}$ of melted löwensteinJensen (LJ) agar and $10 \mathrm{ml}$ of NPs were poured and subsequently, serial dilution was performed. On the following day, $100 \mu \mathrm{l}$ of 10-2 and 10-4 McFarland of bacteria were added and then they are incubated at $37^{\circ} \mathrm{C}$. The colony-forming unit (CFU) of bacteria was counted after 28 days. The atomic force microscopy (AFM) (Institute for color science \& technology, IRAN) of mixture MgONPs-ZnONPs fulfilled by commercial AFM system (Nanosurf, Switzerland) [10]. The TEM image was prepared at the Institute of Biochemistry and Biophysics (IBB) of Tehran University.

\section{MTT assay}

To MTT assay, Vero and $\mathrm{HepG}_{2}$ cell lines (Institute for tuberculosis research, the University of Illinois at Chicago, USA) (1×104 cells per well) were seeded in 96-well plates containing $100 \mu \mathrm{l}$ of DMEM, 
separately [11]. $100 \mu \mathrm{l}$ of MgONPs, ZnONPs, and MgONPs-ZnONPs were inoculated to each well. Next, 5 mg MTT per $\mathrm{ml}$ added. Cell viability calculated using DMSO treated Vero and $\mathrm{HepG}_{2}$ as the $100 \%$ viable control and measured (A540 of NPs $\times$ treated sample/A540 of control) $\times 100$.

\section{Statistical analysis}

Statistical analyses were prepared by using of Kruskal-Wallis test and Mann-Whitney U test. The statistical significance threshold was resolute as P-value $\leq 0.05$.

\section{Results}

The MIC of MgONPs was $0.195 \mu \mathrm{g} \cdot \mathrm{ml}^{-1}$ against $10^{4}$ of $\mathrm{H}_{37} \mathrm{Rv}$ Mtb (Table $1 \mathrm{~A}$ ). According to the results, the MIC of ZnONPs was reported $0.937 \mu \mathrm{g} \cdot \mathrm{ml}^{-1}$ for $10^{-2} \mathrm{H}_{37} \mathrm{Rv}$ Mtb and the MIC of ZnONPs reached to $0.468 \mu \mathrm{g} \cdot \mathrm{ml}^{-1}$ for $10^{-4} \mathrm{H}_{37} \mathrm{Rv}$ Mtb. As well, $0.332 \mu \mathrm{g} \cdot \mathrm{ml}^{-1}$ and $0.166 \mu \mathrm{g} \cdot \mathrm{ml}^{-1}$ of MgONPs-ZnONPs was able to inhibit $10^{-2}$ and $10^{-4} \mathrm{H}_{37} \mathrm{Rv}$ Mtb, respectively (Table $1 \mathrm{~A}$ ). The MIC of MgONPs against both $10^{2}$ and $10^{4}$ concentrations of MDR-Mtb were $12.5 \mu \mathrm{g} \cdot \mathrm{ml}^{-1}$ (Table 1A). The MIC of MgONPs/ZnONPs against $10^{2}$ concentrations of MDR-Mtb was determined $0.166 \mu \mathrm{g} \cdot \mathrm{ml}^{-1}$ (Table $1 \mathrm{~A}$ ). The MIC of MgONPs/ZnONPs against $10^{4}$ concentrations of MDR-Mtb reached to $0.664 \mu \mathrm{g} \cdot \mathrm{ml}^{-1}$ (Table $1 \mathrm{~A}$ ).

The MBCs of MgONPs against $10^{2}$ and $10^{4}$ concentrations of $\mathrm{H}_{37} \mathrm{Rv}$ Mtb were $3.125 \mu \mathrm{g} \cdot \mathrm{ml}^{-1}$ and $1.875 \mu \mathrm{g} \cdot \mathrm{ml}^{-1}$. The MBCs of MgONPs against $10^{2}$ and $10^{4}$ concentrations of MDR-Mtb were $\geq 12.5$ $\mu \mathrm{g} \cdot \mathrm{ml}^{-1}$ (Table 1A). The MBC of ZnONPs was $0.937 \mu \mathrm{g} \cdot \mathrm{ml}^{-1}$ against $10^{4}$ concentrations of $\mathrm{H}_{37} \mathrm{Rv} \mathrm{Mtb}$ (Table 1A). Based on the results, the MBC value of ZnONPs increased to $1.875 \mu \mathrm{g} \cdot \mathrm{ml}^{-1}$ against $10^{-4}$ concentrations of MDR-Mtb. On the other hands, testing showed that the MBCs of MgONPs/ZnONPs reached to $1.328 \mu \mathrm{g} \cdot \mathrm{ml}^{-1}$ against $10^{4}$ concentrations of both $\mathrm{H}_{37} \mathrm{Rv}$ Mtb and MDR-Mtb (Table $1 \mathrm{~A}$ ).

Dose-response curves showed ZnONPs and MgONPs-ZnONPs have high potency to eliminate $10^{-4} \mathrm{MDR}-$ Mtb (Figure 1).

AFM image confirms that the MgONPs-ZnONPs are mono-disperse (Figure 2a). The sizes of the NPs were estimated at less than $50 \mathrm{~nm}$. As we show in Figure 2a, some of NPs attached to the cell wall of Mtb (Figure 2a). It showed that agglomerated metallic NPs are able to attach to the cell wall and penetrates onto Mtb. The oval-shaped Mtb in the present of MgONPs-ZnONPs was demonstrated by TEM images (See also Figure 2b).

MTT assay results showed that MgONPs inhibited the growth of $\mathrm{HepG}_{2}$ cell lines (Table 1B). Also, $3.579 \mu \mathrm{g} . \mathrm{ml}^{-1}$ of $\mathrm{ZnONPs}$ was able to inhibit the growth of $\mathrm{HepG}_{2}$ and Vero cell lines. The results showed 
that the toxicity of mixture MgONPs-ZnONPs reached to $1.233 \mu \mathrm{g} \cdot \mathrm{ml}^{-1}$ against Vero cells. The results indicated the $\mathrm{IC}_{50}$ of $\mathrm{HepG}_{2}$ cell lines in exposure to MgONPs -ZnONPs reached to $0.414 \mu \mathrm{g} \cdot \mathrm{ml}^{-1}$.

\section{Discussion}

The current study is the first experimental study of which has evaluated the MICs and MBCs of ZnONPs, MgONPs, and MgONPs-ZnONPs against $\mathrm{H}_{37} \mathrm{Rv}$ Mtb and the MDR-Mtb. In this study, the toxicity effects of the NPs have evaluated, as well. This study reported the MIC of MgONPs against MDR-Mtb was 12.5 $\mu \mathrm{g} \cdot \mathrm{ml}^{-1}$ (Table 1A). Up to now, there has not been the report in the light of antibacterial effects of MgONPs against Mycobacterium tuberculosis. Previously, Nhu-YThi Nguyen has been reported that MgONPs was able to inhibit gram-positive, gram-negative, and endospore-forming bacteria [12]. Punjabi reported that $1.25 \mu \mathrm{g} . \mathrm{ml}^{-1}$ of ZnONPs inhibited clinical isolated of MDR-TB. 4 Patil et al., reported that ZnONPs has ability to inhibit Mtb at $12.5 \mu \mathrm{g} \cdot \mathrm{ml}^{-1}$ [13]. We showed that the ZnONPs has the ability to eliminate MDR-TB at $1.875 \mu \mathrm{g} \cdot \mathrm{ml}^{-1}$ (Table 1A). Mixture MnO2NPs-ZnONPs also showed bactericidal effects on MDR-TB at $1.328 \mu \mathrm{g} \cdot \mathrm{ml}^{-1}$ the dose-response curves also showed that anti-tubercular impact of NPs depends on the concentration of MDR-TB (Figure 1).

The size of NPs may also affect their anti-tubercular properties. The previous study showed that the ZnONPs with smaller size exhibited higher efficiencies against Mtb [14]. The bigger size of MgONPs about $20 \mathrm{~nm}$ - in comparison with ZnONPs -about $4 \mathrm{~nm}$ - might be effective on weakly anti-tubercular activities of MgONPs. Moreover, researchers believed that metallic NPs have a strong tendency to agglomerate in the broth suspension because of their high surface energy, which could have affected the interactions and penetrations of these nanoparticles with Mtb.

\section{Limitations}

We believe that there are no standards of regular experimental techniques for testing the antitubercular properties of NPs. In fact, NPs with varying sizes, physicochemical characterizes, concentrations, and different initial seeding densities have been used in the previous studies, which do affect the results, significantly. We found that ZnONPs and mixture MgONPs-ZnONPs not only have higher bactericide behavior but might have also synergistic effects against MDR-TB. Actually, even though the current study does prove that $\mathrm{MgO}$ and ZnONPs have anti-tubercular properties against MDRMtb, the anti-tubercular potency of mixture MgONPs/ZnONPs is still unknown and incomparable.

\section{Abbreviations}

Mtb: Mycobacterium tuberculosis

MDR-TB: Multidrug-resistant Mycobacterium tuberculosis

ZnONPs: Zinc oxide nanoparticles 
MgONPs: Magnesium oxide nanoparticles

IC50: The half maximal inhibitory concentration

$\mathrm{HepG}_{2:}$. Human liver cancer cell line

Vero: African green monkey cell line

MICs: Minimum inhibition concentration

LJ: Löwenstein-Jensen agar

CFU: Colony-forming unit

AFM: Atomic force microscopy

IBB: Institute of Biochemistry and Biophysics

\section{Declarations}

\section{Ethics approval and consent to participate}

This study was in accordance with the declaration of Helsinki and an ethical approval was sought from the institutional Ethics Committee of Guilan University of Medical Sciences (Approval No.

IR.GUMS.REC.1396.481). However, because we only used leftovers from clinical specimens, the local ethics committee waived the need for informed consent.

\section{Availability of data and materials}

The datasets used and/or analyzed during the current study are available from the corresponding author on reasonable request.

\section{Funding support}

This study was supported by Guilan University of Medical Sciences. This funding source had no role in the design of this study and will not have any role during its execution, analyses, interpretation of the data, or decision to submit results.

\section{Acknowledgement}

This research supported by vice chancellor of research and technology, Guilan University of Medical Sciences. We thank our colleagues from Guilan University of Medical Siences who provided insight and expertise that greatly assisted the research although they may not agree with all of the interpretations/conclusions of this paper. We would also like to show our gratitude to the Dr. Hadi Sedigh Ebrahim-Saraie for sharing their pearls of wisdom with us during the course of this research, and we 
thank 3 "anonymous" reviewers for their so-called insights. We gratefully acknowledge the dedicated efforts of the investigators and coordinators participated in this study.

\section{Consent for publication}

Not applicable.

\section{Competing interests}

The authors declare that they have no competing interests.

\section{Author's contributions}

M. Shahriari and A. Jafari: conceived the study. M. Shahriari and A. Jafari: participated in the design of the study and performed the statistical analysis. A.A. Foumani and S. Falahatkar: interpreted the data. A. Jafari: obtained ethical clearance and permission for study. S. Falahatkar: Supervised data collectors. F. Movahedzadeh and A. Jafari: Drafting the article or revisiting it critically for important intellectual content. A.A. Foumani: was project leaders and primary investigators of the study. All authors read and approved the final manuscript.

\section{References}

1. Millard, J., C. Ugarte-Gil, and D. Moore, Multidrug resistant tuberculosis. Bmj, 2015. 350: p. h882.

2. Mody, V.V., et al., Introduction to metallic nanoparticles. J. Pharm. Bioallied Sci, 2010. 2(4): p. 282.

3. Nguyen, N.T., et al., Antimicrobial Activities and Mechanisms of Magnesium Oxide Nanoparticles (nMgO) against Pathogenic Bacteria, Yeasts, and Biofilms. 2018. 8(1): p. 16260.

4. Punjabi, K., et al., Efficiency of Biosynthesized Silver and Zinc Nanoparticles Against Multi-Drug Resistant Pathogens. Frontiers in microbiology, 2018. 9.

5. Gholami, M., et al., Nano polyamidoamine-G7 dendrimer synthesis and assessment the antibacterial effect in vitro. Tehran University Medical Journal TUMS Publications, 2016. 74(1): p. 25-35.

6. Soenen, S.J., et al., Cellular toxicity of inorganic nanoparticles: common aspects and guidelines for improved nanotoxicity evaluation. Nano today, 2011. 6(5): p. 446-465.

7. Seddon, J.A., et al., Culture-confirmed multidrug-resistant tuberculosis in children: clinical features, treatment, and outcome. Clin Infect Dis, 2012. 54(2): p. 157-66.

8. Upton, A., et al., In vitro and in vivo activities of the nitroimidazole TBA-354 against Mycobacterium tuberculosis. Antimicrob. Agents Chemother., 2015. 59(1): p. 136-144.

9. Franzblau, S.G., et al., Rapid, low-technology MIC determination with clinical Mycobacterium tuberculosis isolates by using the microplate Alamar Blue assay. J. Clin. Microbiol, 1998. 36(2): p. 362-366.

10. Tomaszewska, E., et al., Detection limits of DLS and UV-Vis spectroscopy in characterization of polydisperse nanoparticles colloids. J Nanomater, 2013. 2013: p. 60. 
11. Mohanty, S., et al., Cationic antimicrobial peptides and biogenic silver nanoparticles kill mycobacteria without eliciting DNA damage and cytotoxicity in mouse macrophages. Antimicrobial agents and chemotherapy, 2013: p. AAC. 02475-12.

12. Nguyen, N.T., et al., Antimicrobial Activities and Mechanisms of Magnesium Oxide Nanoparticles (nMgO) against Pathogenic Bacteria, Yeasts, and Biofilms. Sci Rep, 2018. 8(1): p. 16260.

13. Patil, B.N. and T.C. Taranath, Limonia acidissima L. leaf mediated synthesis of zinc oxide nanoparticles: A potent tool against Mycobacterium tuberculosis. Int J Mycobacteriol, 2016. 5(2): p. 197-204.

14. Jafari, A., et al., Bactericidal impact of Ag, ZnO and mixed AgZnO colloidal nanoparticles on H37Rv Mycobacterium tuberculosis phagocytized by THP-1 cell lines. Microb Pathog, 2017. 110: p. 335-344.

\section{Tables}

Table 1: The MICs, MBCs (A) and MTT assay (B) results of MgONPs, ZnONPs and mixture MgONPs-ZnONPs

(A)

\begin{tabular}{|c|c|c|c|c|c|c|c|c|c|}
\hline \multirow[t]{3}{*}{ Nanoparticle } & \multirow{3}{*}{$\begin{array}{c}\text { Highest concentration } \\
\qquad\left(\mu \mathrm{g} \cdot \mathrm{ml}^{-1}\right)\end{array}$} & \multicolumn{4}{|c|}{$H_{37} R v$} & \multicolumn{4}{|c|}{$M D R-T B$} \\
\hline & & \multicolumn{2}{|c|}{$\begin{array}{c}\text { MIC }(\mu \mathrm{g} \cdot \mathrm{ml} \\
\left(^{-1}\right.\end{array}$} & \multicolumn{2}{|c|}{$\begin{array}{c}\text { MBC }(\mu \mathrm{g} \cdot \mathrm{ml} \\
\left(^{-1}\right.\end{array}$} & \multicolumn{2}{|c|}{$\begin{array}{c}\text { MIC }(\mu \mathrm{g} \cdot \mathrm{ml} \\
\quad\left(^{-1}\right.\end{array}$} & \multicolumn{2}{|c|}{$\begin{array}{c}\text { MBC }(\mu \mathrm{g} \cdot \mathrm{ml} \\
\left(_{-1}^{-1}\right.\end{array}$} \\
\hline & & $10^{-2}$ & $10^{-4}$ & $10^{-2}$ & $10^{-4}$ & $10^{-2}$ & $10^{-4}$ & $10^{-2}$ & $10^{-4}$ \\
\hline $\mathrm{MgO}_{2}$ & 12.5 & 0.390 & 0.195 & 3.125 & 1.875 & 12.5 & 12.5 & $\begin{array}{c}12 . \leq \\
5\end{array}$ & $\begin{array}{c}12 . \leq \\
5\end{array}$ \\
\hline $\mathrm{ZnO}$ & 30 & 0.937 & 0.468 & 7.5 & 0.937 & 1.875 & 0.937 & 7.5 & 1.875 \\
\hline $\begin{array}{c}\mathrm{MgONPs} / \mathrm{ZnO} \\
\mathrm{NPs}\end{array}$ & 42.5 & 0.332 & 0.166 & 2.656 & 1.328 & 1.328 & 0.664 & 5.312 & 1.328 \\
\hline
\end{tabular}

(B) 


\begin{tabular}{|c|c|c|c|c|}
\hline Nanoparticle & $\begin{array}{c}\text { Initial } \\
\text { concentration } \\
\left(\mu \mathrm{g} . \mathrm{ml}^{-1}\right)\end{array}$ & $\begin{array}{l}\text { Stock } \\
(\mu \mathrm{g} \cdot \mathrm{ml} \\
-1)\end{array}$ & $\begin{array}{c}\mathrm{IC}_{50} \text { of } \\
H e p G_{2} \text { cell lines ( } \mu \mathrm{g} \cdot \mathrm{ml} \\
\left.{ }^{-1}\right)\end{array}$ & $\begin{array}{c}\mathrm{IC}_{50} \text { of } \\
\text { Vero cell lines ( } \mu \mathrm{g} \cdot \mathrm{ml} \\
\left.{ }_{-1}^{-1}\right)\end{array}$ \\
\hline $\mathrm{MgO}_{2}$ & 25 & 12.5 & 0.279 & 1.134 \\
\hline $\mathrm{ZnO}$ & 60 & 30 & 3.579 & 3.579 \\
\hline $\begin{array}{c}\mathrm{MgO} \text { NPs/ZnO } \\
\text { NPs }\end{array}$ & 42.5 & 21.25 & 0.414 & 1.233 \\
\hline
\end{tabular}

Figures
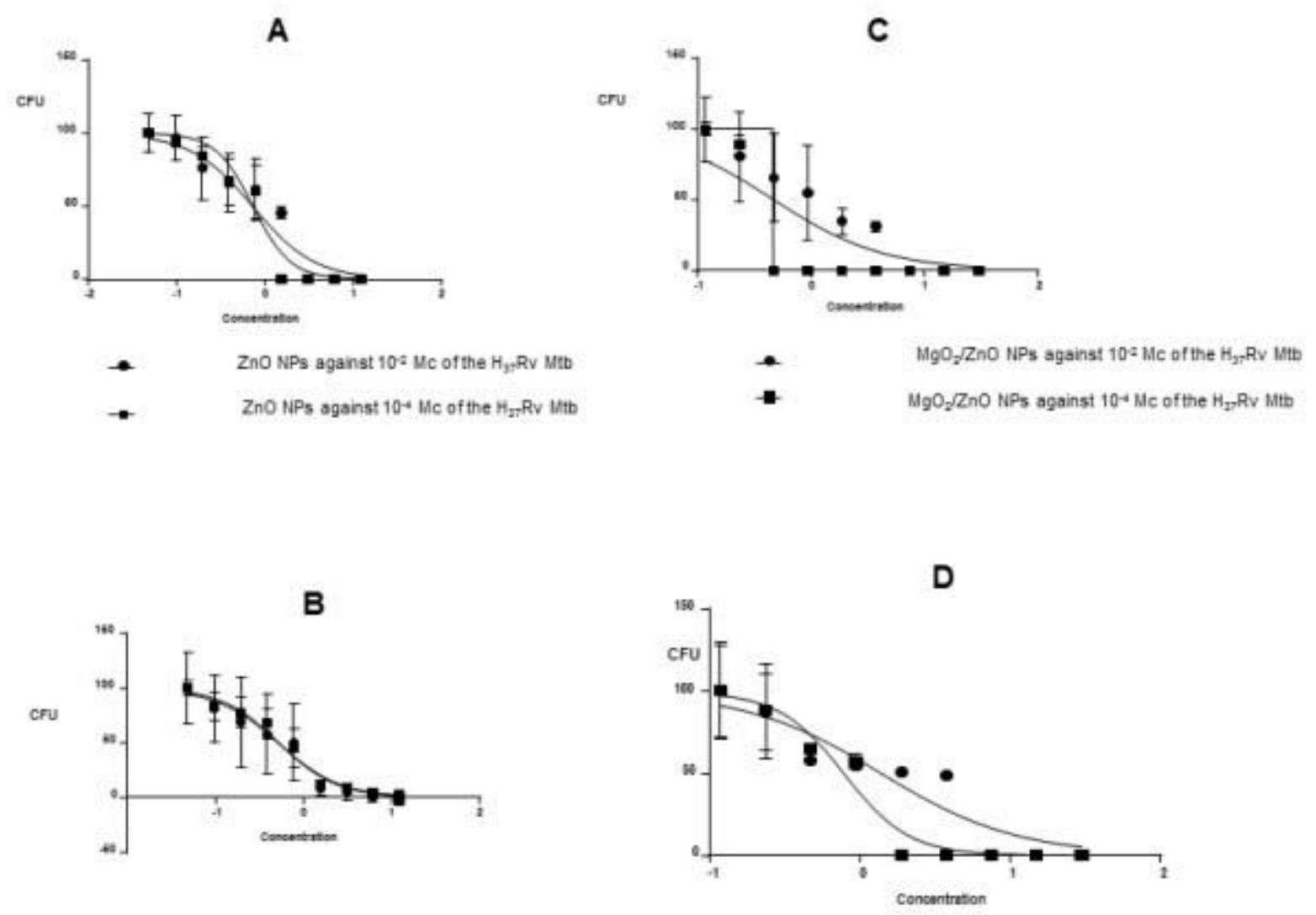

ZnO NPs against 10: Me of the MOR-TB ZnO NPs against $10^{-4}$ Mc of the MOR-TB

$\mathrm{MgO}_{2} \mathrm{Z} \cap \mathrm{O}$ NPs against $10^{2}$ Me of the UDR-TB $\mathrm{MgO}, \mathrm{ZnO}$ NPs against $10^{-4}$ MC of the MDR-TB

\section{Figure 1}

The dose - response curves of ZnONPs against H37Rv Mtb (a) and against MDR-Mtb (b). The dose response curves of MgONPs-ZnONPs against H37Rv Mtb (c) and against MDR-Mtb (d) in 10-2 and 10-4 McFarland. 


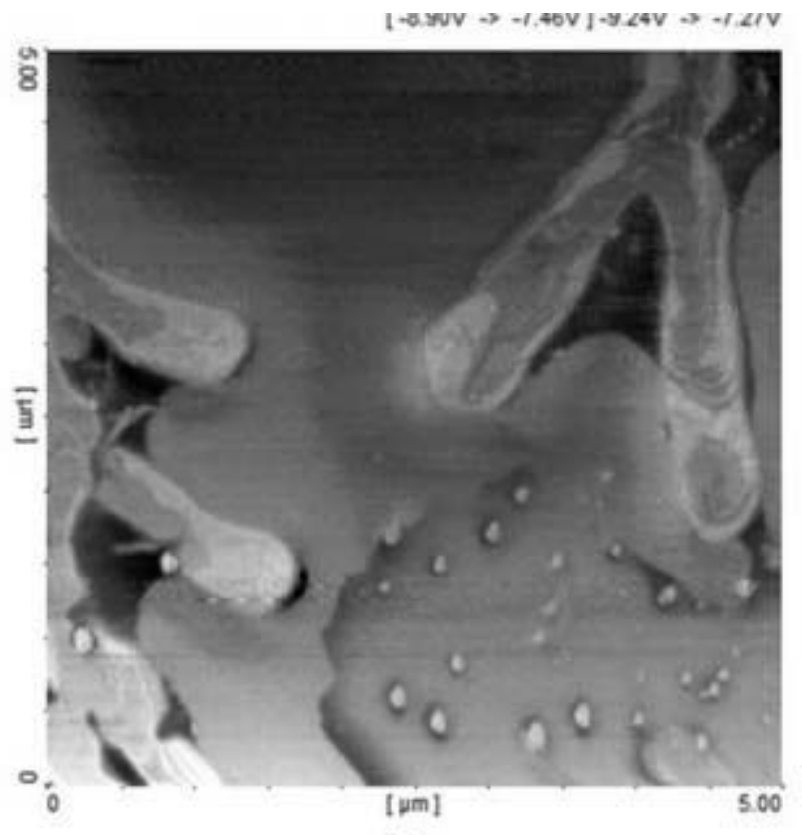

(a)

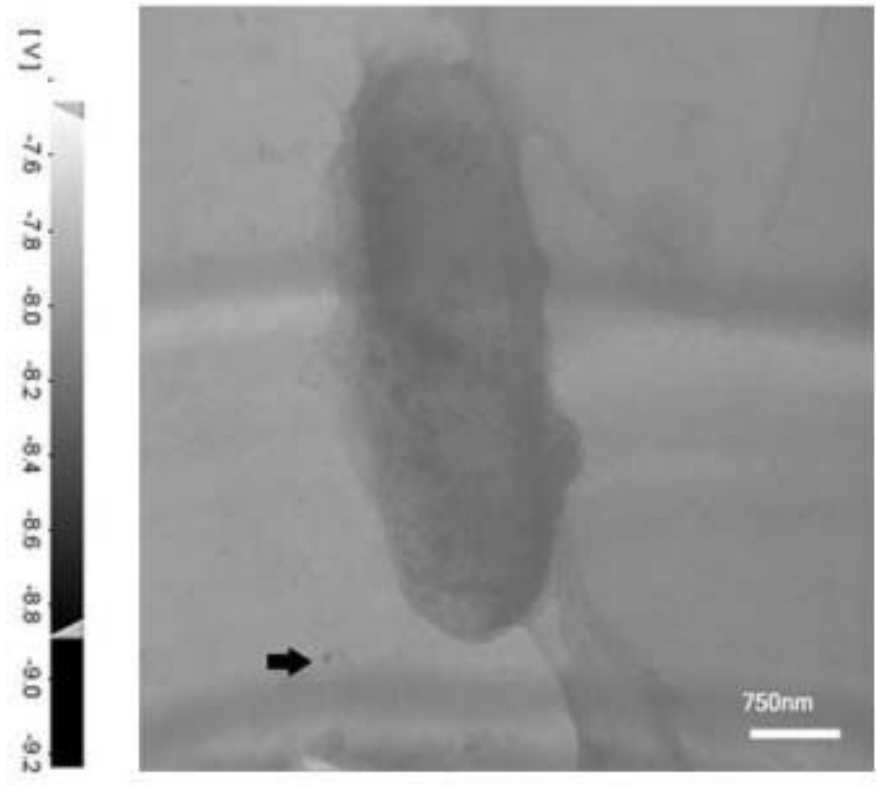

(b)

Figure 2

AFM image of H37Rv Mtb and mixed MgONPs-ZnONPs (a) on silicon surface and TEM image (b) of NPs around of H37Rv Mtb on the scale of $750 \mathrm{~nm}$. 